\title{
LAS RAÍCES AGUSTINIANAS DE LA CONCEPTUALIDAD $D E$ "SER Y TIEMPO"
}

\section{Dante Klocker*}

La progresiva publicación a lo largo de las últimas dos décadas de los cursos dictados por Heidegger durante sus primeros años de docencia ha permitido reconstruir el proceso de gestación de su primera "obra capital", Ser y Tiempo, sus múltiples referencias textuales e influencias. Entre ellas, ocuparía un lugar destacado el pensamiento de San Agustín, por el que Heidegger sentía una alta estima y con el que mantuvo en varias ocasiones un intenso diálogo hermenéutico ${ }^{1}$. Para justificar esta afirmación me propongo mostrar aquí su fuerte presencia en algunos de los conceptos centrales de la célebre obra de 1927. Esta presencia consistirá algunas veces en lo que, a partir de las fuentes con que contamos, puede ser considerado como la huella resultante del trato interpretativo con el texto agustiniano. Otras, en significativas analogías y afinidades entre ambos pensadores que creo posible identificar y mostrar, aún sin estar en condiciones de explicar como resultado de una apropiación expresa. En caso de tratarse de lo primero, caben aún varias posibilidades. Las ideas de Agustín pueden aparecer como fuente si no única, al menos dominante; pueden, en otros casos, producir un efecto convergente junto a otras no menos importantes;

*UCSF-UNL, mpklocker@infovia.com.ar

${ }^{1}$ Estas ocasiones habrían sido, sobre todo, las lecciones dictadas en la Universidad de Friburgo durante el Semestre de Verano de 1921 tituladas Agustín y el neoplatonismo; un curso planeado para el Semestre de Verano de 1924 que desafortunadamente jamás llegaría a dictarse, al menos no de modo público y abierto, sino a lo sumo como "seminatio privatissimun", de lo cual tampoco habría hasta el momento ninguna evidencia (cf. Kisiel, Th., The genesis of Heidegger's "Being and Time", University of California Press, California, 1995, p. 558); una conferencia acerca de la cuestión del tiempo en el libro XI de las Confesiones pronunciada en el convento benedictino de Beuron el 26 de octubre de 1930 y un seminario de tema similar dictado en la Universidad de Friburgo durante el Semestre de Invierno de 1930-31 (cf. Ibid., p. 468). De todos ellos, sólo ha sido editados Agustin y el neoplatonismo en Heidegger, M., Pbänomenologie des religiösen Lebens, Klostermann, Frankfurt a. M., 1995, GA 60, pp. 157-299. 
pueden, finalmente, tener una incidencia tan sólo secundaria, subordinada a otros motivos teóricos o indirecta.

I. "Inquietum est cor nostrum": la "inquietud" o "cuidado" (Sorge) como constitución ontológica fundamental de la subjetividad humana.

El primer concepto a tener en cuenta ( $y$ en torno al cual giran de algún modo todos los pertenecientes a la "analítica existencial" elaborada en Ser y Tiempo) es, sin duda, el de "Sorge" (preocupación, solicitud, cuidado), que caracteriza la constitución ontológica fundamental del existir humano. Heidegger reconoce expresamente la inspiración agustiniana de dicho concepto ${ }^{2}$, cuyos primeros esbozos podrían buscarse en los cursos dictados durante 1919 y 1920. En efecto, según ya se afirmaba en esa época, "Agustín vio en el 'inquietum cor nostrum' la gran e incesante inquietud de la vida", poniendo, así, de manifiesto "un aspecto completamente nuevo" y "originario"3 de ella. Tengamos en cuenta que el Heidegger de estos años intentaba llevar a cabo una investigación a la que comenzaba a llamar "fenomenología hermenéutica", cuya tarea consistía en evitar la distorsión y desfiguración del "fenómeno" ocasionada por la vía teórico-objetivadora para acceder, así, a la esfera preteórica de la "vida fáctica" (es decir, propia, singular, concreta, histórica) y captar sus caracteres esenciales. Y es a partir de este proyecto que él descubre en Agustín a un pensador original y, más aún, "originario" que lo pone en la pista de lo que está buscando. La vida no es sino "inquietud", no entendiendo por esto una especie de estado o proceso "psicológico", sino la determinación ontológica fundamental de la "ejecutividad" vital. La radicalidad alcanzada por Agustín al señalar este rasgo consistiría precisamente en que no lo captó "sólo de modo teórico, sino que vivió en él y lo trajo a expresión"4.

Esta interpretación va a ser confirmada y desarrollada poco después en unas lecciones acerca del libro $\mathrm{X}$ de las Confesiones. En este sentido,

${ }^{2} \mathrm{Al}$ respecto, puede verse la última nota al pie del parágrafo 42 de Ser y Tiempo (Heidegger, M., Sein und Zeit, Klostermann, Frankfurt a. M., GA 2, 1977, p. 264).

3 Heidegger, M., Grundprobleme der Pbänomenologie, Klostermann, Frankfurt a. M., 1993, GA 58, pp. 62 у 205.

4 Ibid., p. 62. 
resulta especialmente relevante prestar atención al análisis heideggeriano del motivo que sirve de hilo conductor a la primera parte de este libro: la búsqueda de Dios, identificada y referida por Agustín a la búsqueda de la vida feliz: "Cuando te busco a tí, Dios mío, es la vida bienaventurada lo que busco. Que te busque yo para que viva mi alma, pues si mi cuerpo vive de $\mathrm{mi}$ alma, mi alma vive de ta"5. Deteniéndose en la significación fenomenológico-existencial de este "buscar" (quaerere), Heidegger comenta lo siguiente: "yo no soy sólo aquel del que parte el buscar para dirigirse a algún lugar, o aquel en el que acaece el buscar, sino la ejecución misma del buscar"6. Éste no consistiría, pues, en un comportamiento particular entre otros (tales como investigar, trabajar, amar, etc.), sino en un carácter ontológico constitutivo de todo comportarse respecto de los objetos. Dicho de otro modo, el yo concreto no sólo (ni fundamentalmente) realiza determinados actos a los que podríamos caracterizar como búsquedas, sino que es el buscar mismo. A su vez este quaerere significa para Agustín Deum qua vitam beatam quaerere. Pero este acusativo tampoco señalaría un mero "contenido" objetivo al que se mantuviera referida un cierto tipo de búsqueda (diferente en este aspecto de aquellas por la que, por ejemplo, se busca trabajo, pareja, etc.). Indicaría, por el contrario, la dirección inherente a todo buscar en cuanto tal, independientemente de cual sea en cada caso su objeto; o, lo que es lo mismo, la dirección esencial de la dinámica tendencial de la vida misma, en cuyo horizonte tiene lugar toda relación con objetos particulares.

En este contexto la vida queda claramente caracterizada como cura (preocupación, cuidado, etc.) ${ }^{7}$ por la vita beata, las cuales constituyen dimensiones inseparables y coesenciales de un mismo fenómeno: la cura sólo es tal en vistas de la vita beata y la vita beata, a su vez, sólo se presenta en y como cura. Y es precisamente este término latino el que Heidegger traducirá al alemán primeramente como "Bekümmerung" y luego por el casi sinónimo de "Sorge", que vemos aparecer en Ser y Tiempo. Afirmará, por ejemplo, ya en

5 San Agustín, Las Confesiones, en Obras de San Agustín (ed. bilingüe), BAC, Madrid, 1955, Tomo II, X, 20, 29.

${ }^{6}$ Heidegger, M., Phänomenologie des religiösen Lebens, p. 192.

7 Heidegger cita en numerosas oportunidades este pasaje de las Enarrationes in Psalmos VII, 9 donde Agustín emplea expresamente dicho término: "Delectatio finis curae". 
1922: "El sentido fundamental de la movilidad vital fáctica es el Sorgen (curare)"8. Ahora bien, este concepto, que señala en los escritos tempranos simplemente el carácter dinámico-ejecutivo de la existencia ${ }^{9}$, aún sin perder del todo esta orientación inicial, tras la reelaboración $\mathrm{y}$, sobre todo, formalización a que será sometido en la obra de 1927, comenzará a referirse a una "totalidad estructural originaria" en la que se articulan tres "modos de ser": a) la "existenciariedad", b) la "facticidad" y c) la "caída"10.

\section{II. "Cada una en todas, ya una en dos, ya dos en una": la "cooriginariedad" de las tres estructuras ontológicas fundamentales.}

a) Resulta clara la contribución de Agustín (en este punto quizás como un exponente más, aunque sobresaliente, de la experiencia cristiana) en lo que respecta a la tematización de la primera. En un pasaje de la Confesiones aparece la distinción entre dos modos de posesión de la vida feliz: están, por un lado, lo "felices en realidad" (re beatr) y, por otro, lo "felices en la esperanza" (spe beati) ${ }^{11}$, es decir, aquellos que gozan ya de la vida celestial y aquellos que, hallândose aún en la vida terrena, viven ya aquí en pos de aquélla. Éstos, aún en la vita praesens (es decir, fáctica e histórica), viven anticipatoriamente en la espera de la vita futura. Para Heidegger esta orientación esencial de la experiencia cristiana hacia el futuro pone de manifiesto un rasgo constitutivo de la experiencia humana en general. Por eso, la afirmación agustiniana según la cual "vuestra vida ha de seguir aquello a lo que se adelanta vuestra esperanza (quo praecedit spes vestra,sequatur vita vestra)", es reinterpretada y reapropiada por Heidegger -claro está por vía de secularización o, si se prefiere, de ontologización- del siguiente modo: "La vida se ejerce en vistas de aquello hacia lo cual se adelanta el esperar

${ }^{8}$ Heidegger, M., "Phänomenologische Interpretationen zu Aristoteles. Anzeige der hermeneutischen Situation" (Natorp-Bericht), en Dilthey Jabrbucb, 6, 1989, p. 240.

9 Véase también, por ejemplo, Heidegger, M., Pbänomenologische Interpretationen $z^{u}$ Arisıteles. Einfübrung in die phänomenologische Forschung, Klostermann, Frankfurt a. M., 1985, $\subset A$ 61, p. 93: "El preocuparse (Sorgen) se ejecuta, la vida fáctica es en la ejecución del preocuparse".

${ }^{10}$ Véase Heic'egger, M., Sein und Zeit, \$41, pp. 254 ss.

11 Cfr. San Agustín, op. cit., X, 20, 29. En realidad, Agustín menciona también un tercer grupo que c: el de "los que no son felices ni en esperanza ni en realidad", pero esta categoría es sencillamente omitida por Heidegger. 
(Erwarten)"12. Esto es, es constitutivo del existir humano un movimiento de anticipación por el que constantemente se proyecta más allá de sí hacia posibilidades propias a partir de las cuales se comprende a sí mismo y a las cosas. Y es precisamente este "adelantar-se" (sich-vorweg-sein) el que es pensado en Sery Tiempo como primer momento estructural de la Sorge.

b) Aún teniendo en cuenta que el origen y la trama de referencias del segundo concepto es compleja ${ }^{13}$ y que no sería posible reconocer una incidencia ni destacada ni directa del pensamiento agustiniano en su acuñación, no resulta, sin embargo, difícil mostrar su cercanía con la noción judeo-cristiana de "creatio". Así como ésta implica haber sido "puesto" (gestellt sein) en el mundo, es decir, "recibir la existencia"14, aquélla alude al "puro" y "desnudo [hecho de] 'que se es y se tiene que ser" "15; es decir, al faktum primordial del existir, situado siempre "ya" en un determinado mundo al que "no se ha traído por sí mismo"16 (es decir, a través de una decisión libre y autónoma), sino al que ha sido "arrojado" (geworfen). De este modo, el sujeto humano "no es el fundamento de su ser", si bien ha sido "despedido (entlassen) por el fundamento" para existir -sólo desde allí y de modo necesario- como fundamento, esto es, como instancia proyectiva y generadora de posibilidades.

Ahora bien, cuando Heidegger se refiere en el parágrafo 29. de Ser y Tiempo al modo primario de acceso del sujeto a esta condición de "arrojado" y a su mundo y afirma (en clara alusión a los límites de la fenomenología husserliana) que éste jamás reside en la "intuición", sino en una forma de captación emotiva y preteórica a la que él llama "encontrarse" (Befindlichkeit), cita en una nota al pie un pasaje tomado del Contra Faustum XXXII, 18,

12 Heidegger, M., Phänomenologie des religiösen Lebens, p. 272. La cita de Agustín pertenece a las Enarrationes in Psalmos CXXXVI, 22.

${ }_{13} \mathrm{Al}$ respecto puede verse el excelente y exhaustivo artículo de Kisiel, Th., "Das Entstehen des Begriffsfeldes 'Faktizität' im Frühwerk Heideggers”, en DiltheyJabrbuch 4 (1986-87), pp. 91-120.

${ }_{14}$ Ibid., p. 249. Acerca de esta conexión puede verse también Kisiel, Th., op. cit., p. 119.

15 Heidegger, M., Sein und Zeit, \29, p. 179. Puede verse también Heidegger, M., Prolegomena zur Geschicbte des Zeitbegriffs, Klostermann, Frankfurt a. M., 1979, GA 20, p. 402.

${ }^{16}$ Heidegger, M., Sein und Zeit, $₫ 58$, p. 377. 
donde Agustín afirma que "no se entra en la verdad, sino por la caridad". Sin duda, está viendo aquí un importante apoyo para su tesis acerca del valor cognoscitivo o "des-cubridor" ( $y$, por otra parte, a su juicio, metodológicamente indispensable) de los fenómenos afectivos.

c) Resulta aún más decisivo el diálogo con Agustín en lo que respecta al tratamiento de la tercera estructura ontológica: la "caída". Ésta es vista ya en el curso de 1921 como rasgo inherente a la cura en cuanto preocupación por la vida feliz. Precisamente porque los hombres constitutivamente tienden a la delectatio, es decir, al goce de la felicidad, fácilmente "caen en lo que pueden y con ello se contentan, porque aquello que no pueden no lo quieren tanto cuanto es menester para poderlo"17. Es decir, el yo por su dinámica propia lleva necesariamente en sí la tendencia a "in multa defluere", a derramarse y perderse en la diversidad de los objetos inmediatamente accesibles en el mundo, quedando allí capturado y clausurado su horizonte vital. Sería, pues, inherente a todo comportamiento existencial un "serarrastrado orientado por y en la delectatio" en cuanto ésta se ve constantemente reclamada por la "multiplicidad significativa" del mundo" Heidegger cree hallar aquí un "cómo del experimentar", es decir, una determinación esencial de la totalidad de la experiencia humana, a partir de la cual traduce en clave ontológico-existencial una noción propiamente religiosa: la de "tentación". La afirmación "vita temptatio tota"19 significará ahora que la tentación no es sino un rasgo constitutivo universal de la vida fáctica ${ }^{20}$.

Según el comentario agustiniano a la Primera Carta de San Juan 2, 15 17, esta tendencia existencial a la dispersión (defluxio) y absorción en el mundo tendría lugar en tres direcciones fundamentales. En la primera, la "concupiscencia de la carne" (concupiscentia carnis), de lo que se trata es, pues, de cómo tiene lugar la referencia más inmediata del yo al "mundo circundante" (Um-w velt). Éste no se presenta primariamente con carácter de mero "objeto sensible" (lo cual supondría ya un primer distanciamiento

17 San Agustín, Las Confesiones X, 23, 33, pp. 507. Las cursivas son mías.

${ }^{18} \mathrm{Cfr}$. Heidegger, M., Phänomenologie des religiösen Lebens, p. 206.

${ }^{19}$ San Agustín, op. cit., X, 32, 48, p. 520.

20 Esta noción reaparecerá expresamente en 1927 para señalar uno de los rasgos pertenecientes al fenómeno de la "caída", junto con el carácter tranquilizador, alienante, etc. Cfr. Heidegger, M., Sein und Zeit, §38, pp. 233 ss. 
respecto de un "sujeto" que se limita a fijar y constatar lo que tiene delante), sino como aquello de lo que se ocupa (usando, evitando, manipulando, consumiendo, etc.) el "trato" (Umgehen) fruitivo. La tentación reside aquí en el peligro para el yo de quedar cautivo en la incitación de todo aquello que presenta un atractivo "sensual", sin conseguir situarlo dentro de una perspectiva vital más amplia; es decir, en "dejar a un lado las posibilidades y fortificarse en lo real, en lo [inmediatamente] significativo"21. Sin embargo, hay una segunda forma de disfrute, vinculada como las anteriores a la actividad de los sentidos, pero que por sus características propias no puede ser asimilada a aquéllas. El deseo (cupiditas) ya no apuntará aquí a "deleitarse en la carne (in carne)", sino a "experimentar a través de la carne (per carnem)"22. Es decir, la finalidad del deseo ya no consiste en la posesión fruitiva de las cosas, sino en la "vana y curiosa" contemplación de las mismas a través de la experiencia. Correlativamente su objeto deja de ser también sólo lo agradable (lo bello, armonioso, suave, sabroso, etc.) para incluir también aquello que desde el punto de vista de la "concupiscencia de la carne" sería desagradable y hasta insoportable (Agustín menciona, por ejemplo, el placer de contemplar cadáveres despedazados). Este "apetito de conocer" es llamado por Agustín "concupiscencia de los ojos" (concupiscentia oculorum) en referencia a que éstos son, a su juicio, si no los únicos al menos sí sus principales agentes.

En su análisis fenomenológico Heidegger insiste en que aquí estaríamos ante un nuevo acto (es decir, una nueva forma de intencionalidad) en la cual "de lo que se trata propiamente no es del contenido, sino de la 'relación' y, por cierto, de la mera ejecución de la relación"23. Es decir, ha desaparecido el trato directo y activo en el cual el placer se hallaba centrado en el disfrute de los objetos mismos. Éstos han sido, ahora, puestos "a distancia" de modo tal que el goce en lugar de recaer prioritariamente sobre ellos, lo hace más bien sobre el acto mismo que los intenciona. Y éste consiste simplemente en "ver", en el sentido de "mero mirar hacia o considerar" 24 . El yo, que en la primera forma de "concupiscencia" se perdía a sí mismo en lo que comparecía como

${ }^{21}$ Heidegger, M., Pbänomenologie des religiösen Lebens, pp. 218-19.

${ }^{22}$ San Agustín, op. cit., X, 35, 54, p. 528. Las cursivas son mías.

${ }^{23}$ Heidegger, M., op. cit., pp. 223-24.

${ }^{24}$ Cfr. Ibid., p. 226. 
placentero, ahora lo hace en lo que se presenta como novedoso, interesante, llamativo, etc. desde una curiosidad siempre voluble e insatịsfecha ${ }^{25}$.

En ambos casos "el existir (Dasein), el sí-mismo es un quedar absorbido (Aufgehen). El sí-mismo es vivido por el mundo"26. En ambos casos, además, este "mundo" hacia el cual el existir se precipita es el "mundo-circundante" (Umwelt). Pero la "caída" puede tener lugar también en dirección al "mundo-de-la-convivencia" (Mitwelt) con otros sujetos ante cuya mirada el yo intenta "hacerse valer", tanto en la búsqueda de estima y reconocimiento ("amari velle", querer ser amado), como en el deseo de dominación y sometimiento del otro ("timeri velle", querer ser temido) ${ }^{27}$. Este "querer ser temido y amado por los hombres no por otra cosa, sino por conseguir de ello un goce"28, en cuanto tercera modalidad de la caída será denominada por Agustín "ambición del siglo". En ella es el mundo propio (las propias cualidades, logros, capacidades, etc.) el que aspira a pasar a un primer plano y ser tenido como significativo y valioso y lo hace buscando una posición destacada o preponderante en el mundo compartido. Pero, de este modo, no consigue sino "verse en los ojos, en las pretensiones, en el gusto o disgusto, en las veleidades y en la tontería de los otros"29. Es decir, la comprensión y valoración de sí queda alienada en la mirada ajena de la

25 Estas interpretaciones en torno a Confesiones X, 35 serán retomados en el parágrafo 36 de Ser y Tiempo donde se analiza este fenómeno de la "curiosidad" o "avidez de novedades" (Neugierigkeit) como una de las manifestaciones fundamentales del "caída", junto a la "habladuría" y la "ambigüedad".

${ }^{26}$ Heidegger, M., op. cit., p. 228.

27 En clara continuidad, a mi juicio, con estos análisis en el parágrafo 26 de Ser y Tiempo, se caracteriza al modo inauténtico y cadente de realización de la referencia a los demás sujetos como "sustitutivo-dominante", en cuanto se sustrae al otro la iniciativa y decisión de sus acciones en el "mundo" (esto es, su "Sorge"), ocupando (es decir, usurpando) su lugar. Frente a esto, se propone como relación genuina y propia la llamada "anticipativo-liberadora", que se adelanta al otro no para arrebatarle su libertad, sino para permitirle ejercerla, promoviendo su ser-sí-mismo. Sería posible, quizás, advertir como trasfondo de esta segunda noción la idea agustiniana expresada en su "Amo: volo ut sis" ("Amo: quiero que seas"), tantas veces mencionada por Heidegger.

${ }^{28}$ San Agustín, op. cit., X, 36, 59, p. 532.

${ }^{29}$ Heidegger, M., op. cit., p. 229. 
que se espera aprobación o sumisión. Con esto el yo sucumbe al poder del mundo compartido y, una vez más, abjura de sí y se pierde.

En Ser y Tiempo la noción de "caída" va a quedar fijada en términos similares como aquel estado existencial en el que el Dasein humano se halla "inmediata y regularmente" (zunächst und zumeist) y que consiste en un estar "perdido" y "absorbido" por el "mundo" cotidiano y público de la ocupación. El modo de interpretación aquí dominante consiste en que nada es comprendido a partir de un descubrir genuino y debidamente legitimado, sino sólo sobre la base del anónimo "se" (Man) presente en lo que "se" dice, "se" piensa, "se" considera correcto, etc. Este "se" expropia al yo sustrayéndole la conducción de su propia vida. Éste ya no comprende sus posibilidades vitales desde sí, sino desde lo otro de sí, quedando, de este modo, alienado o enajenado (Entrremdung). Ahora bien, este fenómeno, presentado aquí en términos antropológico-existenciales (es decir, como un carácter perteneciente a la vida humana en general) conlleva importantes consecuencias para un tipo particular de actividad como es la reflexión filosófica. En ella el mencionado movimiento de caída en el mundo se manifiesta como tendencia del sujeto reflexionante a interpretar su propio ser (y, más aún, el "ser" en general) tomando como orientación y referencia el ser de los entes con los que constantemente trata en el mundo (y esto es precisamente lo que Heidegger critica a la ontología tradicional). Es posible advertir una curiosa coincidencia entre este análisis de la persistente dificultad del yo para alcanzar una genuina autocomprensión y el siguiente pasaje del De Trinitate:

¿Qué hay tan presente al alma como el alma? Pero como se apega a las cosas en que piensa con amor y está familiarizada por el afecto con los objetos sensibles o corpóreos, no es capaz de pensar en sí misma sin las imágenes de dichos objetos. De ahí nace su bastardía errónea, pues no puede separar de sí los fantasmas de lo sensible y verse por sí sola ${ }^{30}$.

Y si bien este texto no es -hasta donde sé- jamás citado por Heidegger en favor de su propia interpretación del existir, la primera mención a Agustín realizada en Ser y Tiempo guarda una relación directa con esta cuestión. Se cita allí el siguiente pasaje de las Confesiones. "Ciertamente

30 San Agustín, Tratado sobre la Santísima Trinidad, en Obras de San Agustín (ed. bilingüe), BAC, Madrid, 1948, Tomo V, X, 8, 11, pp. 505. 
trabajo en ello y trabajo en mí mismo, y me he hecho a mí mismo tierra de dificultad y de excesivo sudor" 31 . Esta dificultad -que, como se vio, tendría su fundamento en la constitución misma del Dasein humano, en su tendencia al autoencubrimiento, propia de la "caída"- se manifestaría no sólo en la "opacidad óntica" del yo (por ejemplo, la que tiene que ver con la captación de sus propios estados psicológicos), sino también y fundamentalmente en su "opacidad ontológica", es decir, aquella vinculada a la comprensión de sus estructuras de "ser". En este sentido, y en términos que recuerdan la paradoja expresada en el texto recién citado del De Trinitate, afirmará Heidegger que "lo ónticamente más cercano y conocido es lo ontológicamente más lejano, desconocido y constantemente pasado por alto"32.

Estos tres momentos estructurales de la Sorge -existenciariedad, facticidad y caída - en cuanto constitución del existir humano mantienen entre sí una relación a la que Heidegger llama de "cooriginariedad" (Gleichursprünglichkeit). Esto quiere decir, en primer lugar, que no hay entre ellas una "conexión de fundamentación" (como sería el caso si alguna de ellas se asentara o fundara en otra que constituyera su condición de posibilidad), sino que se mueven en el mismo nivel de radicalidad ontológica. Esto hace que cada una de ellas en su irreductible diversidad pero así también en su inescindible unidad respecto de las demás las atraviese y determine, siendo a su vez y recíprocamente atravesada y determinada por ellas. La proyectualidad, por ejemplo, está siempre situada (o "arrojada") y, a la inversa, la propia situación fáctica es en sí misma proyectante. Este modelo explicativo presenta, a mi juicio, una. sorprendente semejanza con el propuesto por Agustín en el De Trinitate acerca de la estructura del alma. Allí para aclarar la peculiar relación existente entre "mente", "conocimiento" y "amor" o entre "memoria", "inteligencia" y "voluntad" afirma que "si bien cada una es en sí misma singular, mutuamente se hallan todas en todas, ya una en dos, ya dos en una"33. Agustín ilustra esta paradoja (reflejo para él del misterio trinitario) de que la parte posea la misma extensión que el todo sin dejar por eso de ser parte del siguiente modo: "recuerdo que poseo

${ }^{31}$ San Agustín, Las Confesiones X, 16, 25, p. 495.

${ }^{32}$ Heidegger, M., Sein und Zeit, \$9, 2, p. 59.

${ }^{33}$ San Agustín, Tratado sobre la Santísima Trinidad IX, 5, 8. 
memoria, entendimiento y voluntad; comprendo que entiendo, quiero y recuerdo; quiero querer recordar y entender"34.

\section{III. "Tempus distentio animi": la temporalidad como fundamento de la constitución ontológica de la subjetividad humana.}

Hemos llegado, entonces, hasta aquí, a la aclaración del fenómeno internamente múltiple y articulado de "facticidad", "existenciariedad" y "caída" como momentos co-constituyentes de la "totalidad originaria" de la "Sorge". Este punto de llegada es esencial, pero, con todo, insuficiente, ya que es aún necesario preguntar por el fundamento que da unidad a este todo estructural. Y éste residirá en la "temporalidad" (Zeitlicbkeit):

El fundamento ontológico originario de la existencialidad del Dasein es, pues, la temporalidad. Sólo desde ella resulta existencialmente comprensible la totalidad estructural articulada del ser del Dasein en cuanto Sorge [... Las estructuras ontológicas del Dasein ya alcanzadas tienen que ser retrospectivamente puestas al descubierto en vistas de su sentido temporal ${ }^{35}$.

Es decir, la "existenciariedad" deberá ser reinterpretada a partir de su enraizamiento en el éxtasis temporal del "futuro o porvenir", la "facticidad" en el "sido" y la "caída" en el "presentar". De este modo y siguiendo una estricta "relación de fundamentación" que determina el ritmo interno de la obra se pasa de la primera sección titulada "Etapa preparatoria del análisis fundamental del Dasein" a la segunda, decisiva y culminante (al menos en lo que respecta a la "analítica existenciaria"), "Dasein y temporalidad".

Heidegger mismo ha llamado la atención acerca del paralelismo existente entre esta transición desde la dilucidación del fenómeno de la "Sorge" al de la "temporalidad" como su condición última de posibilidad y el nexo -a su juicio, esencial- que une el libro X y el libro XI de las Confesiones. Mientras "la pregunta más íntima de los libros I-X" habría sido "quid est bomo", ésta da "paso" (Übergang) en el libro siguiente a la cuestión "quid est tempus" en la que "las Confessiones alcanzan su auténtica profundidad y, en

\footnotetext{
${ }^{34}$ Ibid., X, 11, 18.

${ }^{35}$ Heidegger, M., op. cit., \45, pp. 311-12.
} 
esta profundidad radical, su máximo alcance"36. Aquí Agustín presenta su concepción del tiempo como "distentio animi" y, por tanto, podríamos decir a la inversa del "animus" (esto es, de la subjetividad humana) como "distentio temporalis", como acaecer temporal ${ }^{37}$.

\section{La "verdad interior" como "llamada" a la existencia auténtica.}

Pero retornemos nuevamente al fenómeno de la "caída", que tratábamos más arriba. Como veíamos, éste ya había sido tematizado en el curso sobre Agustín, donde se afirmaba que es inherente a la cura por la vita beata la temptatio a quedar absorbido en los distintos polos de significatividad del mundo (los objetos de disfrute, la mirada de los otros, etc.). Ahora bien, conjuntamente con esta tendencia a perderse en una "existencia inauténtica" pertenece también a la cura como posibilidad esencial el "contramovimiento" hacia la superación del estado de caída, es decir, hacia la recuperación de sí. Porque aún en medio de la inautenticidad opera el "tenue recuerdo"38 de la "verdad", que no cesa de convocar al sujeto a una vida según ella. Esta incitación interior es presentada por Agustín a través de la metáfora visual de la luz (lumen, luz del alma, diferente de lux en cuanto mera luz física) y la auditiva de la voz interior. Es posible, a mi juicio, reconocer las huellas de este célebre motivo agustiniano (sobre el que Heidegger meditó detenidamente en el curso de 1921) con lo que en Ser y Tiempo será la "Ilamada" de la "conciencia" por la cual el Dasein es convocado desde sí mismo y, sin embargo, al mismo tiempo desde "más allá" de sí a una existencia genuina y "propia" 39.

Por otra parte, Heidegger se refiere también a una experiencia privilegiada capaz de "aislar" o "individualizar" de modo eminente al Dasein

36 Estas afirmaciones proceden de una conferencia aún inédita pronunciada en Beurón bajo el título de "Consideraciones de San Agustín sobre el tiempo. Confessiones lib. $X T$ ”, folio 11. Agradezco especialmente al Lic. Mario M. Gómez de la UBA por haberme facilitado una copia de este texto.

37 Acerca de esta nueva analogía entre los dos filósofos puede verse, por ejemplo, von Herrmann, F.-W., 'Die 'Confessionnes' des Heiligen Augustinus im Denken Heideggers", en Quaestio I/2001, Heidegger e i medievali, a cura di Esposito, C. e Porro, P., Brepols/Pagina, Turnhout/Bari, 2001, p. 129.

${ }^{38}$ Cfr. San Agustín, Las Confesiones X, 23, 33, p. 507.

${ }^{39}$ Cfr. Heidegger, M., op. cit., $\ 57$, pp. 364 ss. 
en cada caso propio arrancándolo de su estado de caída en la publicidad del "se": la angustia. Este temple anímico es cuidadosamente distinguido del "miedo" con el que se corre el riesgo de confundirlo. Mientras éste surge siempre ante un objeto del mundo identificado como una amenaza, el antequé de la angustia, en cambio, no es ningún ente determinado, por lo cual parece no estar en ninguna parte y, más aún, no ser "nada". La razón de esto es que aquello que nos angustia y desazona no es sino la totalidad de nuestro "ser-en-el-mundo" y la posibilidad "cierta" e "irrebasable" de "ya-no-sermás-en-el-mundo", es decir, la muerte. En una nota al pie hacia el final del parágrafo 40 de Ser y Tiempo donde se comienza a tematizar este fenómeno existencial, Heidegger remite como un importante antecedente suyo a la distinción agustiniana entre "timor castus" y "timor servilis"40 que ya había tenido oportunidad de analizar en el curso de 1921.

La diferencia entre éstos reside en que, mientras el último se da cuando se busca a Dios sólo por temor a las penas que, en caso de no hacerlo, podrían sobrevenir al yo, el primero consiste en buscarlo por él mismo y en temer en esa medida su pérdida definitiva ${ }^{41}$. Es decir, en un caso se ama porque se teme y en el otro se teme porque se ama; en un caso se desea el bien por él mismo y en el otro sólo para evitar un mal. Éste es para Agustín el "temor del mundo", es decir, leído heideggerianamente, un temor experimentado sobre la base de nuestra relación pragmática con los objetos del mundo; el otro, en cambio, es el "temor puro", en cuanto en él no está en juego el beneficio o perjuicio ocasionado por determinados entes, sino la totalidad de nuestra existencia concreta y fáctica puesta ante la posibilidad de su propia perdición (para Heidegger quizás la única experiencia que permite ganarse viviendo genuinamente).

40 Es importante recordar que en el mismo lugar Heidegger reconoce también su evidente deuda con el pensamiento de Kierkegaard. No obstante, un reconocido estudioso de esta etapa de la obra heideggeriana llega a afirmar que la influencia agustiniana en este punto habría sido tanto o más decisiva que la ejercida por el pensador danés (cfr. Kisiel, Th., "Heidegger reads Augustine on fear and trembling", en University of Dayton Review, Volume 22, №3, Summer 1994, p. 298). ${ }^{41}$ Cfr. Heidegger, M., Pbänomenologie des religiösen Lebens, pp. 293 ss. 


\section{El "verbum internum" como paradigma para la captación conceptual de los caracteres ontológicos del sí-mismo.}

Ahora bien, abandonemos por un momento el nivel de análisis seguido hasta aquí -concretamente la consideración teórica orientada a un determinado objeto temático (en este caso las determinaciones ontológicas de la existencia humana) - y volvamos la atención hacia el teorizar mismo. Este plano meta-filosófico o meta-teórico (e incluso podríamos decir de epistemología de la filosofía) fue ampliamente frecuentado y desarrollado en los escritos tempranos que venimos analizando. Allí aparece con insistencia la preocupación por determinar con precisión el estatuto de la conceptualidad filosófica, como así también por establecer sus condiciones de validez, es decir, sus modos propios de verificación o, dicho husserlianamente, de "legitimación" (Ausweisung). Este problema es motivado - con y contra Husserl- por la exigencia típicamente fenomenológica de fidelidad "a las cosas mismas" y podríamos formularlo como sigue: ¿de qué modo se podría captar y expresar conceptualmente este peculiar campo temático que es la vida fáctica sin traicionar su naturaleza propia, es decir, evitando toda forma de "objetivación" o, lo que es lo mismo, de "cosificación"? La propuesta heideggeriana consistirá en distinguir rigurosamente entre aquellos conceptos cuya función consiste en reflejar las propiedades objetivas de los entes del mundo y aquellos que, por el contrario, están orientados a interpretar los distintos caracteres de la "ejecutividad" del existir humano. Mientras los primeros tienen una función meramente representativa y ordenadora de las distintas regiones de objetos dados, los segundos contienen sólo la "indicación formal" (formale Anzeige) de un "camino" a ser seguido y cumplido por la experiencia hermenéutica. Los primeros son característicos de las llamadas "ciencias especiales", los últimos, de la reflexión filosófica.

$\mathrm{Si}$ bien los posibles antecedentes de esta concepción acerca del lenguaje filosófico son múltiples y complejos (la distinción del mismo Husserl entre "generalización" y "formalización" 42 , quizás las reflexiones de Kierkegaard 43 en torno a una forma de pensamiento capaz de captar la "existencia" sin quitarle su carácter de "realidad" reduciéndola a mera

${ }^{42}$ Cfr. Heideggger, M., op. cit., pp. 57 ss.

${ }^{43}$ Cfr. Heidegger, M., Ontologie. Hermeneutik der Faktizität, GA 63, Klostermann, Frankfurt a. M., 1988, p. 30. 
"posibilidad", etc.), en uno de los lugares en que es tratada la cuestión aparece una sugerente mención al pensamiento de Agustín que merece ser tenida en cuenta. Allí se afirma simplemente que el $\lambda o ́$ jo $\varsigma$ de la fenomenología en cuanto expresión categorial y explicativa de los "fenómenos" del existir debe ser entendido en el sentido del "verbum internum" 44 agustiniano, es decir, de la "palabra" que el alma engendra desde y para sí y a través de la cual obtiene y expresa el conocimiento que tiene de sí. Justamente en coincidencia con la intención heideggeriana, este saberse y decirse no es en absoluto una autoobjetivación, sino una "noticia" íntima y amorosa del yo, idéntica con su propio "ser" 45 .

VI. "Si te busco ya te he encontrado": la búsqueda de Dios como modelo estructural para la elaboración de la pregunta por el "ser".

Para finalizar este recorrido retornemos al punto donde lo iniciamos -el concepto de Sorge como respuesta al problema acerca de la constitución fundamental del existir humano- y recordemos lo que motiva en Ser $y$ Tiempo este problema. De lo que se trata en esta obra es, tal como Heidegger afirma repetidas veces, de llegar a plantear y elaborar adecuadamente la pregunta por el "ser en general". La "vía" propuesta para ello será la aclaración de la constitución ontológica de aquel ente privilegiado en y por el cual se hace patente el "ser", es decir, el Dasein humano. Este retroceso desde la pregunta por el ser a la pregunta por la subjetividad como lugar de su mostración, recuerda el camino seguido en los primeros capítulos del libro $\mathrm{X}$ de las Confesiones. Allí la búsqueda de Dios se inicia interrogando en vano a las realidades externas (en lenguaje heideggeriano, a los "entes intramundanos"). Pero todas ellas (la tierra, los abismos, el mar, los astros, etc.) responden que allí no se halla lo buscado, ante lo cual Agustín propone pasar a la exploración de aquel que hasta el momento había planteado las preguntas e interpretado las respuestas: el yo "interior". La búsqueda del objeto ha conducido, pues, aquí también al problema acerca de la estructura de aquel que busca. Dios en cuanto objeto al que se aspira deberá ser, por un lado, externo y trascendente respecto al yo (en caso contrario no sería "deseado"), pero al mismo tiempo deberá hallarse de algún modo en la

44 Cfr. Heidegger, M., op. cit., p. 63.

45 Para este concepto puede verse, por ejemplo, San Agustín, Tratado sobre la Santísima Trinidad LX, 7-12, pp. 559 ss. 
"memoria" del sujeto (en caso contrario éste carecería de toda indicación orientadora de la búsqueda). De modo semejante (no en un sentido temático, sino justamente estructural) se afirmará al comienzo de Ser y Tiempo que la pregunta por el ser (como, por otra parte, cualquier pregunta), "en cuanto buscar necesita de una previa conducción a partir de lo buscado"46. Y ésta será proporcionada por una "precomprensión" aún vaga y preconceptual del "ser" en la que "ya siempre" nos movemos, así como, para Agustín, en un cierto conocimiento de la felicidad.

\section{Resumen}

La progresiva publicación a lo largo de las últimas dos décadas de los cursos dictados por Heidegger durante sus primeros años de docencia ha permitido reconstruir el proceso de gestación de Ser y Tiempo (1927), sus múltiples referencias textuales e influencias. Entre ellas, ocupa un lugar destacado el pensamiento agustiniano, por lo cual me propongo considerar aquí su fuerte presencia en algunos de los conceptos centrales de dicha obra.

$\mathrm{El}$ primero a tener en cuenta es el de "Sorge" (preocupación, cuidado) con el que Heidegger caracteriza la constitución ontológica fundamental del existir humano. Sus primeros esbozos habían surgido de la reflexión en torno al célebre "inquietum cor nostrum" y a la cura por la vita beata de Confesiones X. A partir de este mismo texto Heidegger había realizado también una interpretación fenomenológica de la tentación, que constituye una de las primeras elaboraciones de la llamada "caídd", precisamente uno de los momentos estructurales de la "Sorge".

Referencias explícitas o sugerentes analogías entre ambos pensadores pueden apreciarse también entre la explicación de Confesiones XI del "tempu" como "distentio animi" y la tesis heideggeriana de la "temporalidad" como fundamento de la constitución de la subjetividad humana; el tránsito desde la búsqueda de Dios a la exploración de la interioridad de aquel que lo busca y el retroceso desde la pregunta por el "ser" a la pregunta por el que comprende el "ser", entre otros casos no menos significativos.

Palabras clave: "Heidegger", “Agustín”, "fenomenología”, "hermenéutica”, “ser", "existencia". 


\begin{abstract}
The progressive publication -along the past two decades- of the courses taught by Heidegger in his first years in teaching has allowed the reconstruction of the process of creation of Being and Time (1927), its multiple textual references and influences. Among these, St. Augustine's thought bears a prominent place, for which reason I intend to consider its noticeable presence in some of the key concepts in the work mentioned.

The first to be considered is Sorge (concern, care), with which Heidegger characterises the fundamental ontological constitution of human existence. His first drafts emerged from the reflection upon the famous "inquietum cor nostrum" and the cura (care) for the vita beata from Confessions X. Based on this same text Heidegger had offered a phenomenological interpretation of temptation, which constitutes one of the first elaborations of the so-called "fall", precisely one of the structural moments of the Songe.

Explicit references or suggestive analogies between both thinkers can also be appreciated between the explanation of "tempus" as "distentio animi" in Confessions XI and the heideggerian thesis of "temporality" as the basis of the constitution of human subjectivity; the road from the search of God to the exploration of the interiority of the one who looks for Him and the way back from the question about the "being" to the question about the one who understands the "being", in other no less significant cases.
\end{abstract}

Key words: "Heidegger", “Augustine”, "phenomenology", "hermeneutics", "being”, "existence". 